\title{
A Novel Capturing Method for Quantification of Extra-Cellular Nanovesicles
}

\author{
Qu Yin ${ }^{1,2}$, Zunfeng Liü ${ }^{1,2}$, Fabrice Laroche ${ }^{3}$, Xiang Zhou ${ }^{2,4}$, Ningning Shao ${ }^{1,2}$, Bencai Lin ${ }^{1}$, \\ Run Wang ${ }^{1,2}$, Ningyi Yuan ${ }^{1}$, Jianning Ding ${ }^{1, *}$, and Jan Pieter Abrahams ${ }^{5, *}$ \\ ${ }^{1}$ Jiangsu Collaborative Innovation Center of Photovoltaic Science and Engineering, School of Petrochemical Engineering, \\ Department of Materials Science and Engineering, Changzhou University, Changzhou 213164, China \\ 2 Jiangnan Graphene Research Institute, Changzhou 213149, China \\ ${ }^{3}$ Department of Pharmacology and Experimental Therapeutics, The Center for Cancer Research, Section of Hematology and \\ Medical Oncology, Boston University School of Medicine, Boston, MA 02118, USA \\ ${ }^{4}$ State Key Laboratory of Natural Medicines, Department of Organic Chemistry, China Pharmaceutical University, \\ Nanjing 210009, China \\ ${ }^{5}$ C-CINA, Biozentrum Basel, Switzerland; LBR, Paul Scherrer Institute, Villigen, CH-4003, Switzerland
}

\begin{abstract}
Extracellular vesicles (EVs), secreted by cells and found in body fluids play important roles in intercellular communication. Therefore, EVs are receiving increasing attention as potential biomarkers in the diagnosis and prognosis of various diseases. However, the detection and the quantification of EVs are hampered by the nanometer scale of these particles and by the lack of optimized quantification methods. Atomic force microscopy (AFM) is a powerful technology that can detect small particles. Here we report a 3D capture method for sample preparation of AFM which improves the accuracy, sensitivity and reproducibility for EVs' detection, compared to conventional sample preparation methods. By shaking a mica plate in EV solution, all the EVs were captured onto the 2D surface. The majority of the captured particles have a size ranging from 10 to $120 \mathrm{~nm}$, which correlates with size data obtained from transmission electron microscopy studies. This novel sample preparation method has high adaptability potential and can also be applied to other organic and inorganic nanoparticles.
\end{abstract}

Keywords: Extracellular Vesicle, 3D Capture Method, AFM, Quantification, TEM.

\section{INTRODUCTION}

Extracellular vesicles (EVs) are phospholipid spherical structures enclosed by lipid bilayers, and containing proteins, nucleic acids and other cellular component. Cells release EVs through exocytosis. ${ }^{1}$ Several distinct types of EVs were reported in recent studies, including exosomes, micro-vesicles (also called micro-particles), membrane particles, and apoptotic vesicles. ${ }^{2}$ EVs are concentrated carriers of genetic (RNAs) and proteomic information, and thus are increasingly recognized as key players in intercellular communication. ${ }^{3-6}$ Therefore, EVs are receiving increased attention as potential biomarkers in the diagnosis and prognosis of diseases, ${ }^{7}$ and are creating excitement in the field of drug delivery. ${ }^{8-10}$

EVs can be released from cells in response to activation or by apoptosis. For example, circulating EVs are best

\footnotetext{
*Authors to whom correspondence should be addressed.
}

known for binding coagulation factors and exposing tissue factor. ${ }^{11}$ Their absence is associated with a bleeding tendency, and their (increased) presence is associated with disseminated intravascular coagulation and thrombosis. ${ }^{12}$ Elevation in plasma levels of EVs has been described in numerous clinical conditions, most of which also associated with an increased thrombotic risk. ${ }^{13}$ Particularly, EVs have been found to be increased in both solid and hematological malignancies, cardiovascular diseases, systemic inflammatory disease, thrombosis, cancer, sicklecell disease, pre-eclampsia (PE), diabetes mellitus and infections. ${ }^{14-16}$

Elevated numbers of tissue-factor-bearing EVs were found to be one of the causes of cancer-associated thrombosis. Increased levels of endothelial cell EVs are found during acute and chronic phases of thrombotic thrombocytopenic purpura. ${ }^{17}$ Pericardial blood collected at the end of cardiopulmonary bypass was found to contain significantly 
more EVs than blood from the systemic circulation. ${ }^{18}$ It was reported that hypertension was associated with platelet activation and increased EV levels compared to normal individuals with a correlation between EV levels and blood pressure. ${ }^{19}$

It has been found that both platelet and endothelial EVs are increased in number in women with a normal pregnancy compared with nonpregnant healthy women. ${ }^{20}$ It was found that a major proportion of women with miscarriages have elevated endothelial cell EVs, suggesting that a clinical relevance of elevated EV concentration with pregnancy loss. ${ }^{20}$ Women with PE showed increased number in EVs compared with normal healthy pregnant women, including increases in total number of the EVs and their sub-populations. ${ }^{21}$

It is now well recognized that EVs act as vectors of bioactive molecules playing a role in blood coagulation, inflammation, cell activation, and the spread of cancer. Their role both as markers and pathogenic effectors in thrombosis, inflammation and cancer has increased the interest of their measurement in clinical practice. ${ }^{22}$

As the levels of EVs are increased in several diseases and disorders, the utility of measuring EVs as a diagnostic and prognostic marker is currently a subject of intense investigation. ${ }^{12}$ Clinically relevant properties of EVs are size, concentration, morphology, biochemical composition, and cellular origin. A general consensus was reached that EV counts are useful for identifying patients at risk and for monitoring response to treatment. ${ }^{23}$ From the size information of individual EVs, a relative size distribution can be obtained, providing insights into the number of EVs of one particular size relative to those of another size. Therefore, obtaining the EVs' concentration and size distribution is also of substantial clinical relevance. ${ }^{8-10}$

Several techniques have been developed for detecting and quantifying EV levels in circulating blood, such as Fluorescence Automatic Counter and Sorter (FACS), capture-based assays, and Atomic Force Microscopy (AFM) and combinations of these techniques have been previously used for EVs' quantification. ${ }^{12}$ However, EV, detection, quantitation and sizing have been problematic because their size is below the reach of conventional detection methods. ${ }^{24} \mathrm{EV}$ studies are therefore hampered by the lack of accurate detection and quantification methods. ${ }^{25}$

FACS is a fluorescence based technology to quantify micro-sized particles such as cells. This technique requires labeled EVs, with laser light that excites the fluorophore label. Unlabeled EVs are challenging to detect and to quantify. Furthermore, EVs having sizes far below the wavelength of the laser light are hard to detected via FACS. ${ }^{26}$ Capture-based assays allow high-throughput assessment of pro-coagulant features of EVs, ${ }^{27}$ but these assays are not providing information on the size and on the number of EVs. Nanoparticle Tracking Analysis (NTA) allows detection of particles smaller than $50 \mathrm{~nm}$ by tracking the movement of each particle through image tracking analysis. However, the presence of bigger particles potentially limits the detection of smaller particles. ${ }^{28}$

Using AFM, Yuana and collaborators were able to detect EVs as small as $5 \mathrm{~nm} .{ }^{26}$ To this aim, they incubated a drop of solution containing Tissue Factor (TF)-bearing EVs on mica coated with anti-TF antibody, and quantified EVs 1000 fold more effectively than using flow cytometry. ${ }^{26}$ The capturing of EVs on the mica surface relies on Brownian motion, which is very slow and the capture process is quite inefficient, as schematically shown in Figure 1(b). By pipetting the solution up and down over the mica substrate, the capture process could be made more effective up to a limited extent. Nevertheless, many EVs are lost during this sample preparation procedure. Moreover, the experiments are time consuming and poorly reproducible. To improve the EV capture, a microfluidic capturing technique was developed, which pumps the EV solution across the mica surface. This can speed up the capturing process and improves the capture efficiency. ${ }^{29}$ However, the method requires a complicated microfluidic set up and does not fully solve the problem of capturing EVs from the solution to the 2D surface, because the contact of the solution with the $2 \mathrm{D}$ surface is still limited due to laminar flow.

In this paper, we discuss a new, straightforward EV capturing method for AFM sample preparation. We show that shaking a small mica plate in the EV solution, the contact between liquid with the $2 \mathrm{D}$ surfaces of mica is greatly improved compared to the conventional incubation method, as schematically shown in Figure 1(a). In this way, we capture all the EVs very efficiently on both sides of the mica.

\section{EXPERIMENTAL DETAILS}

\subsection{Preparation of EVs}

EVs were isolated from the supernatant of the HEK293T cells, which were cultured according to ATCC recommendations in Dulbecco's Modified Eagle Medium (DMEM) supplemented with fetal calf serum (FCS) and Penicillin Streptomycin mixture (pen/strep). The HEK293T cells were seeded at a confluence of $20 \%$ and cultured

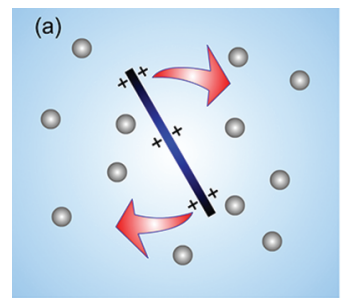

(b)

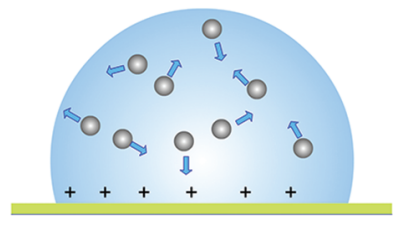

Figure 1. Schematic representation of EV capture from solution onto the positively charged mica (a) by shaking a small mica plate in solution, the EV particles in the 3D solution can be captured on the 2D surfaces of the mica very efficiently. We show this method to be significantly more effective than (b) the conventional incubation technique, which relies on the Brownian motion of the EVs. 


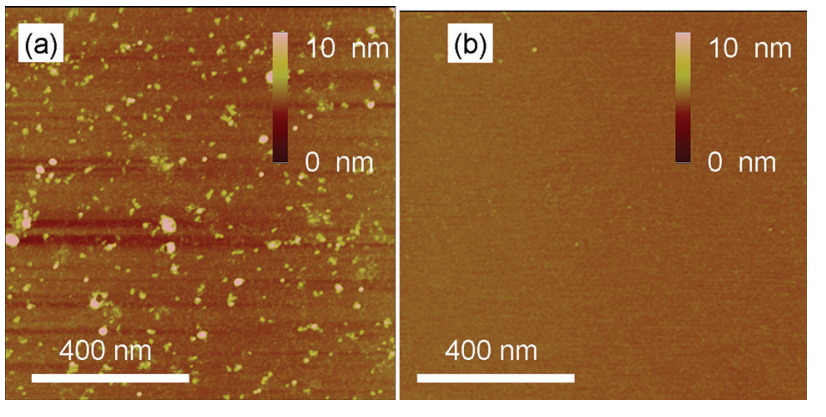

Figure 2. AFM images of the captured EVs using our 3D capturing method from EV solution (20 times dilution): first capture (a), second capture (b).

in $\mathrm{CO}_{2}(5 \%)$ and $95 \%$ humidity at $37{ }^{\circ} \mathrm{C}$ for exactly 72 hours before collecting the supernatant. EVs were isolated from the supernatant by serial centrifugation, which is an adapted version of a protocol from the literature [30].

The protocol of EV isolation was as follows:

(1) Transfer $25 \mathrm{ml}$ of the culture supernatant to $50 \mathrm{ml}$ centrifuge tubes, followed by centrifugation at $300 \mathrm{~g}, 30 \mathrm{~min}$.

(2) $17.5 \mathrm{ml}$ of the supernatant was collected, and then centrifugated at $2000 \mathrm{~g}, 30 \mathrm{~min}$.

(3) $12 \mathrm{ml}$ of the supernatant was collected, then centrifugated at $10.000 \mathrm{~g}, 30 \mathrm{~min}$.

(4) $8.5 \mathrm{ml}$ of the supernatant was collected and transferred, from each of the two equilibrated tubes, to a single aluminum ultracentrifuge tube, so the total volume per ultracentrifuge tube was $17 \mathrm{ml}$.
(5) Ultracentrifugation, $100.000 \mathrm{~g}, 70 \mathrm{~min}$. Supernatant was discarded and the pellet re-suspended in $20 \mathrm{ml}$ PBS.

(6) Ultracentrifugation, $100.000 \mathrm{~g}, 70 \mathrm{~min}$. Supernatant was discarded and the pellet re-suspended in $20 \mathrm{ml}$ PBS in an Eppendorf tube by pipetting up and down and vortexing. The EVs solution was collected at the bottom of the tube by short centrifugation at $500 \mathrm{~g}$ for 15 seconds.

(7) The concentrated EV solution was aliquoted.

(8) EVs aliquots were frozen at $-80{ }^{\circ} \mathrm{C}$ and stored for later use.

\subsection{Protein Content Analysis, Particle Size and Zeta Potential by Dynamic Light Scattering}

The protein content of the isolated EV samples was quantified using a Bradford assay. The particle size distribution and zeta potential of EVs were obtained on a zeta sizer nano series (Nano S) ZEN 1600 (Malven Inc.) by using $100 \mu \mathrm{l}$ of EV solution.

\subsection{The 3D Capture Method for Sample Preparation and Characterisation of EVs by AFM}

Positively charged mica (+mica) plate (diameter: $3 \mathrm{~mm}$ ) was prepared as follows. First, (3-aminopropyl)triethoxysilane (APTES, $30 \mathrm{ml}$ ) was 1:1 diluted with $\mathrm{H}_{2} \mathrm{O}(30 \mathrm{ml})$. The solution was shaken for $20 \mathrm{~s}$ and poured into a petri-dish containing freshly cleaved mica. The mica was incubated in the petri-dish for 2.5 min, and washed with ethanol and $\mathrm{H}_{2} \mathrm{O}$ three times.
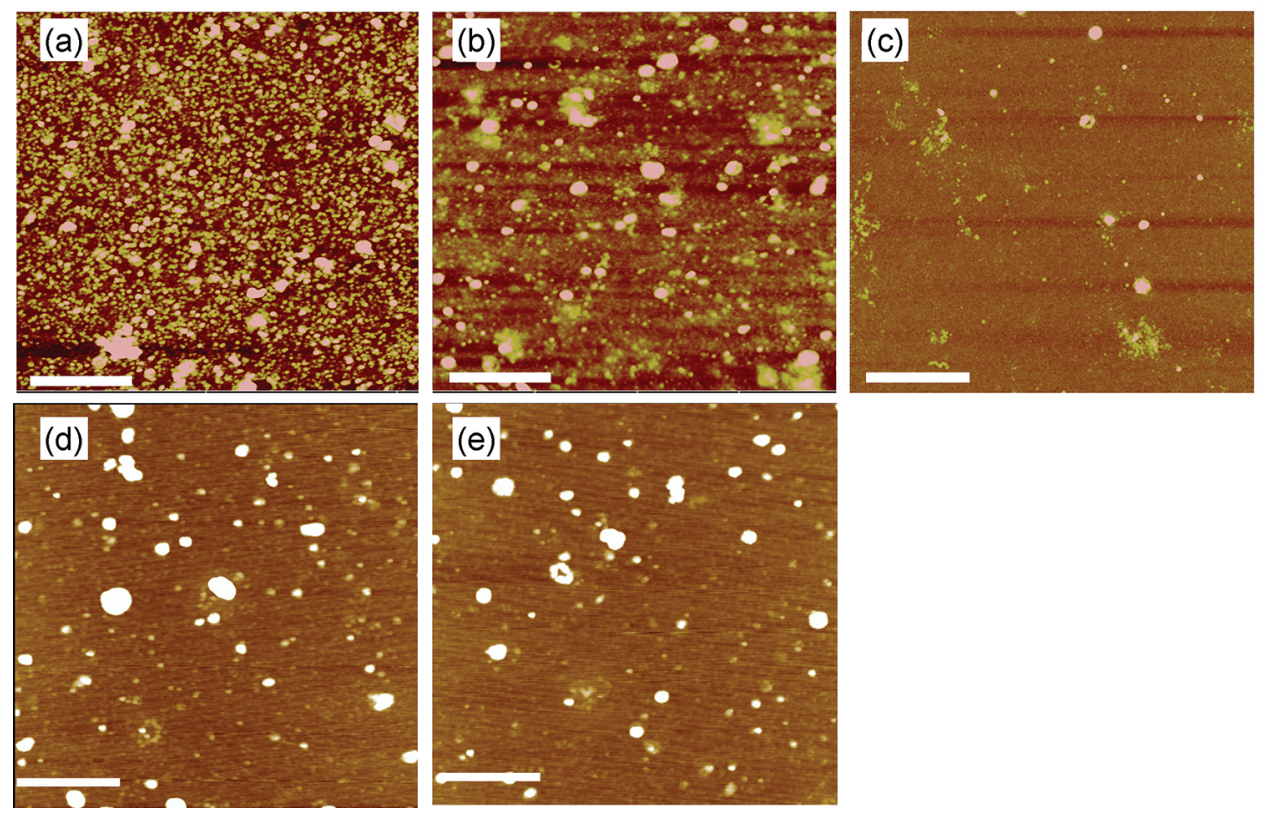

Figure 3. (a-c) AFM images of EVs sequentially captured from a non-diluted solution on positively charged mica via the 3D capturing method ((a) the first + mica incubation, (b) the second + mica incubation, (c) the third incubation of the same sample, shaking time: 10 min), (d) AFM image of EVs captured from non-diluted solution on positively charged mica via the conventional incubation method (incubation time: 30 min); (e) AFM image of EVs captured from non-diluted EV solution on positively charged mica via the conventional incubation method, a pipet was used to mix the EV solution during the incubation (incubation time: $30 \mathrm{~min}$ ); Scale bar: $500 \mathrm{~nm}, \mathrm{Z}$ scale: $10 \mathrm{~nm}$. 

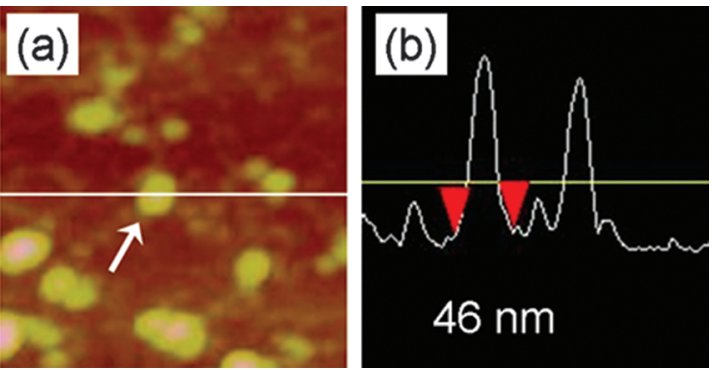

Figure 4. Single EV diameter estimated from the cross-section profile of the AFM data. (a) AFM image, the white arrow indicates the EV of which the diameter was measured across the line; (b) cross-section analysis of the EV diameter, the distance between the two triangles was measured as the diameter.

The +mica plate was put into an Eppendorf tube $(1.5 \mathrm{ml})$ containing EV solution $(50 \mu \mathrm{l})$ and shaken by inverting the Eppendorf tube upside down to allow the mica plate to roll up and down in solution for $10 \mathrm{~min}$. The negatively charged EVs were captured on the + mica. The + mica was washed with MilliQ $\mathrm{H}_{2} \mathrm{O}$ and used for AFM measurements.

Tapping mode AFM (Digital Instruments Dimension 3100 ) at $300 \mathrm{~Hz}$ was used to acquire the AFM images under ambient conditions at a scanning rate of $1 \mathrm{~Hz}$ and a scan angle of $0^{\circ}$ with low free amplitude $(0.6-0.7 \mathrm{~V})$. Images were flattened and plane-fitted except when otherwise stated. The diameter of the AFM tip was approximately 10 to $20 \mathrm{~nm}$. All experiments were repeated in triplicate.

\subsection{The Calculation Process of EV Concentration}

The detailed calculation of EV concentration is as follows:

$$
\begin{gathered}
n=2 \cdot n^{\prime} \cdot S / S^{\prime} \\
C=X \cdot(n / V) \\
S=3.14 \cdot(d / 2) \cdot 2
\end{gathered}
$$

where $d$ is the diameter of the mica disk.

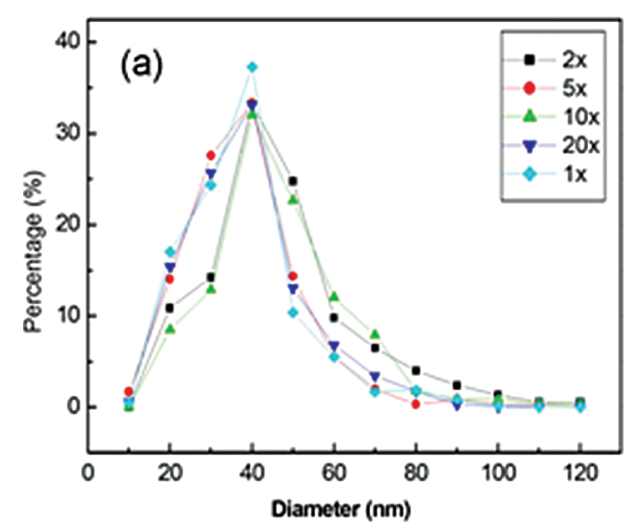

Where $n$ is the total number of EVs in the EV solution, $S$ is the total surface area of mica, $n^{\prime}$ is the EV number on the scanned area, $S^{\prime}$ is the scanned area. The factor " 2 " is required because EVs are captured on both sides of the + mica. $V$ is the total volume of the EV solution, $C$ is the $\mathrm{EV}$ concentration in EV solution, $X$ signifies the dilution.

\subsection{Characterisation by Transmission Electron Microscopy (TEM)}

EVs $(10 \mu 1)$ were mixed with PFA $(10 \mu 1,4 \%)$ for $10 \mathrm{~min}$, and the formvar TEM grid (200 mesh) was put into this mixture $(20 \mu \mathrm{l})$ and shaken for 10 minutes. Then the grid was washed with Phosphotungstic acid (PTA, 1\%) solution, and blotted with filter paper. TEM images were obtained using a JEOL JEM-1010 electron microscope, with an acceleration voltage of $80 \mathrm{kV}$ in vacuum.

\section{RESULTS AND DISCUSSION}

EVs were isolated from the supernatant of HEK293T cells cultured for 72 hours, according to a modified reference method. ${ }^{30}$ In this process, soluble proteins secreted from the cells were removed by serial ultracentrifugation. We obtained $100 \mu \mathrm{l}$ of EVs from $25 \mathrm{ml}$ of cell culture supernatant. The protein content of the isolated EVs was quantified as $35 \mathrm{ng} \mu \mathrm{l}^{-1}$ using Bradford assay. The zeta potential was measured to be $-13 \pm 4 \mathrm{mV}$, indicating the negatively charged nature of the EVs. We were not able to capture these EVs on pristine mica, which is negatively charged (data not shown). Therefore we used positively charged mica to capture the negatively charged EVs via charge interaction.

The +mica (diameter: $3 \mathrm{~mm}$ ) was put into the EV solution and shaken to capture all the EVs on mica after inverting the Eppendorf tube upside-down, as schematically described in Figure 1(a). Tapping mode AFM was used to detect the EVs. In order to verify if all the EVs in solution were captured, this process was repeated with new + mica plates until no EVs could be detected anymore

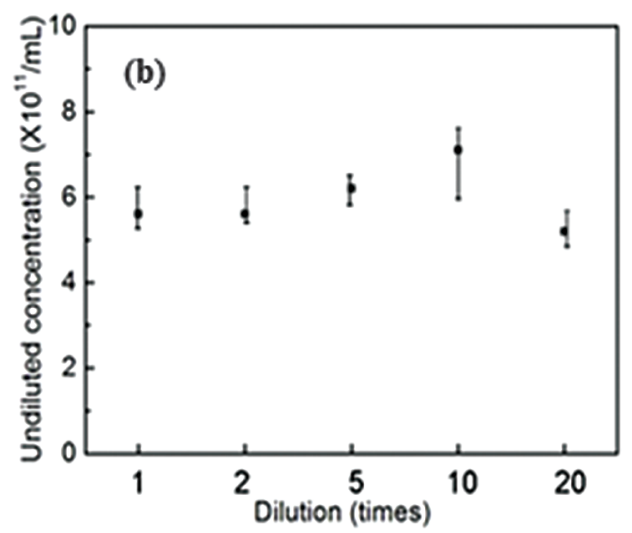

Figure 5. (a) EV diameter distribution measured from different dilutions (1 to 20 times dilution), 300 EVs were counted for every dilution; (b) EV concentration relative to the undiluted solution, measured at different dilutions. 


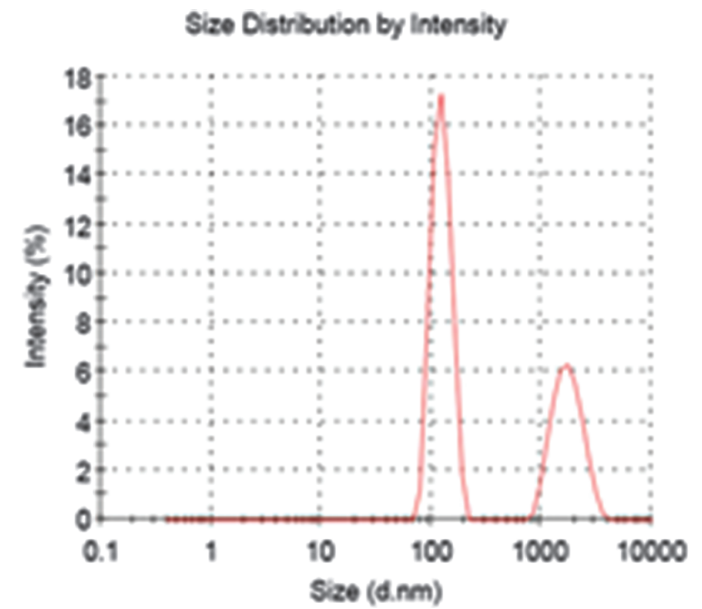

Figure 6. Dynamic light scattering (DLS) analysis of the EV's diameter distribution.

on the + mica, as shown in Figure 2(b). Two mica plates were required to capture all the EVs from the undiluted EV solution, as the 3rd mica plate almost captured no spherical particles, as shown in Figure 3(c). In order to check whether all of the EVs were captured on the mica plate by this $3 \mathrm{D}$ capture method, the EV solution was diluted by 2, 5, 10, and 20 times. For these dilutions, only one mica plate was required to capture all the EVs, as the 2nd mica plate did not capture EVs for these dilutions (see Fig. 2 as an example of 20 times dilution).

Clearly, all the EVs were captured efficiently on the +mica surfaces using our 3D capturing method. Our method is far more efficient than the conventional incubation technique. For comparison, we incubated the undiluted EV solution with + mica for 30 minutes; the results were shown in Figures 3(d and e). Only few EVs were captured, and pipetting the EV solution for mixing did drastically increase the capturing efficiency.

We quantified EVs and measured the size distribution of the EVs on AFM images interactively. The EV diameter can be obtained directly from the "cross-section analysis" function of the AFM software "Nanoscope," as shown in
Figure 4. The EV diameter was measured from the bottom of the cross-section profile, $46 \mathrm{~nm}$ in this case, Figure 4(b). Close to $300 \mathrm{EVs}$ were counted in different locations and in different images from different dilutions. The EV diameter distributions are shown in Figure 5(a). The observed diameter ranges from 10 to $120 \mathrm{~nm}$ and peaked at 40 to $60 \mathrm{~nm}$ for all dilutions. Smaller sized EVs were obtained at the higher dilution rates $(10 \times$ and $20 \times)$ compared to the lower dilutions $(1 \times$ and $2 \times)$, which might be caused by the formation of EV aggregates at higher concentrations.

As all of the EVs from solution $(50 \mu \mathrm{l})$ could be captured (on both sides of the mica), the quantity of the EVs on the scanned area of the mica could be related to the total number of EVs in the starting solution, as described in the Methods Section 2.4. For the case of in Figure 2, the EV solution was diluted by 20 times, so $X=20$. We measured $n^{\prime}=646, S=3.14 \cdot(3000 / 2) 2\left(\mathrm{~m}^{2}\right), S^{\prime}=2.37 \times$ $2.37\left(\mathrm{~m}^{2}\right), V=0.05 \mathrm{ml}$, so we get a concentration of: $C=5.7 \times 1011$ (unit $/ \mathrm{ml})$.

We found that different dilutions produced consistent EV concentrations for the undiluted solution: $\sim 5-6 \times$ $10^{11}$ per $\mathrm{ml}$ (shown in Fig. 5(b)). This indicated that our $3 \mathrm{D}$ capturing method allows reliable quantification of $\mathrm{EVs}$ for different dilutions.

The average EV diameter as suggested by AFM was smaller than the diameter suggested by dynamic light scattering (DLS), which showed one major peak at $120 \mathrm{~nm}$ and a minor peak at $1100 \mathrm{~nm}$, as indicated in Figure 6. The peak at $1100 \mathrm{~nm}$ may be caused by aggregation of the EVs in solution, which is likely to be rare because such aggregation of particles were not observed in the AFM images. But such aggregates will show up in DLS, as they scatter much more strongly than the smaller particles.

The EVs were also characterized by Transmission Electron Microscopy (TEM). The TEM pictures were taken after fixing and staining, as shown in Figure 7(a). The size distribution also ranges between $10-120 \mathrm{~nm}$ and peaked at $40-60 \mathrm{~nm}$, in accordance with the AFM results. Perhaps the DLS measurement suggested a larger size compared to the AFM and TEM because the solution was
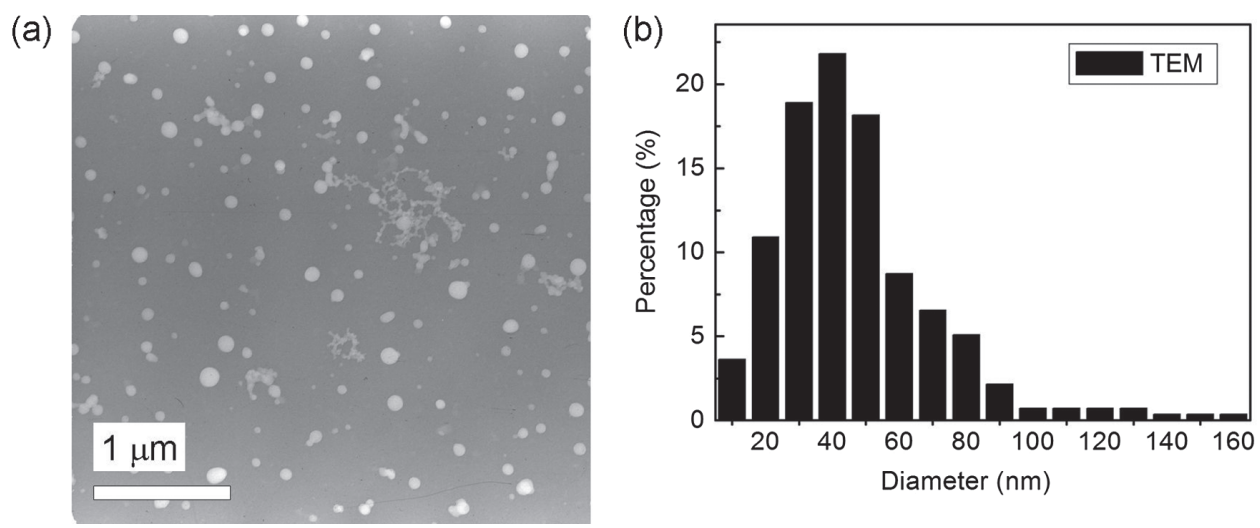

Figure 7. (a) TEM image of EVs fixed by PFA and positively stained with PTA, (b) size distribution of the EVs from TEM images. 
slightly more viscous than the solution used for calibration, due to small (lipid) contaminants. Furthermore, the DLS method measures the hydro-dynamic diameter of the EVs, which should be bigger than the direct microscopic observations. ${ }^{26,29}$ It is also likely that $\mathrm{H}_{2} \mathrm{O}$ evaporated out of the EVs during the AFM imaging and TEM sample preparation, resulting in under-estimation of the EVs' size. However, we did not observe any size decrease during the repeated AFM scanning process.

\section{CONCLUSIONS}

Here, we have demonstrated that by mechanically stirring a mica plate in EV solution, all the EVs could be captured on the mica with high uniformity. The method is very reproducible and is independent of size distribution and concentration of the EVs (up to the saturation point of the mica). Our method significantly reduced the sample preparation for AFM localizing and scanning. As the EV solution can be diluted, very small amounts of EV solution are sufficient to quantify and determine the size distribution and the shape of the EVs. By providing an efficient and reliable sample preparation method, the new method will speed up the detection and quantification of the captured EVs by AFM, and accelerate the application of EVs as potential biomarkers in the diagnosis and prognosis of diseases. In future experiments, the characterization of the subsets of EVs bearing different factors, such as glycoprotein CD42a, phosphatidylserine, and others, ${ }^{17,31}$ by using antigen-specific monoclonal antibodies will be addressed, possibly directly from biological fluids.

Moreover, this 3D capturing method is not only limited to EVs. Also other nanoparticles, such as liposomes, micelles, metal nanoparticles, and other organic and inorganic nanoparticles can also be captured and quantified by choosing suitable affinity matrixes. The accurate quantification of the nanoparticles will benefit several different fields including disease diagnosis, drug delivery, cellular sensors, and etc.

Acknowledgments: The authors gratefully acknowledge the National Natural Science Foundation of China (Grants Nos. 31200637, 51303017), and Jiangsu Basic Rearch Program grant BK2012148. We thank Rodinde Hendrickx, Maxim E. Kuil, Lammers Geda and Federica Galli for technical support.

\section{References and Notes}

1. G. Rapos and W. Stoorvogel, J. Cell Biol. 200, 373 (2013).

2. Y. Yuana, A. Sturk, and R. Nieuwland, Blood Rev. 27, 31 (2013).
3. C. Thery, L. Duban, E. Segura, P. Veron, O. Lantz, and S. Amigorena, Nat. Immunol. 3, 1156 (2002).

4. K. Al-Nedawi, B. Meehan, R. S. Kerbel, A. C. Allison, and J. Rak, P. Natl. Acad. Sci. USA 106, 3794 (2009).

5. M. Baj-Krzyworzeka, M. Majka, D. Pratico, J. Ratajczak, G. Vilaire, J. Kijowski, R. Reca, A. Janowska-Wieczorek, and M. Z. Ratajczak, Exp. Hematol. 30, 450 (2002).

6. T. A. Tian, Y. Y. Wang, H. T. Wang, Z. Q. Zhu, and Z. D. Xiao, J. Cell. Biochem. 111, 488 (2010).

7. A. Piccin, W. G. Murphy, and O. P. Smith, Blood Rev. 21, 157 (2007).

8. L. Varez-Erviti, Y. Q. Seow, H. F. Yin, C. Betts, S. Lakhal, and M. J. A. Wood, Nat. Biotechnol. 29, 341 (2011).

9. E. Van der Pol, A. N. Boing, P. Harrison, A. Sturk, and R. Nieuwland, Pharmaco. Rev. 64, 676 (2012).

10. J. G. Van den Boorn, M. Schlee, C. Coch, and G. Hartmann, Nat. Biotechnol. 29, 325 (2011).

11. R. Lacroix, S. Robert, P. Poncelet, and F. Dignat-George, Semin. Thromb. Hemost. 36, 807 (2010).

12. E. Van der Pol, A. G. Hoekstra, A. Sturk, C. Otto, T. G. Van Leeuwen, and R. Nieuwland, J. Thromb. Haemost. 8, 2596 (2010).

13. A. Falanga, C. J. Tartari, and M. Marchetti, Thromb. Res. 129, S132 (2012).

14. B. Furie and B. C. Furie, Blood cells Mol. Dis. 36, 177 (2006)

15. Y. S. Lee, S. H. Kim, J. A. Cho, and C. W. Kim, Exp. Mol. Med. 43, 281 (2011).

16. L. Burnier, P. Fontana, B. R. Kwak, and A. Angelillo-Scherrer, J. Thromb. Haemost. 101, 439 (2009).

17. J. G. Kelton, T. E. Warkentin, C. P. Hayward, W. G. Murphy, and J. C. Moore, Blood 80, 2246 (1992).

18. R. Nieuwland, R. J. Berckmans, R. C. Rotteveel-Eijkman, K. N. Maquelin, K. J. Roozendaal, P. G. Jansen, K. Ten Have, L. Eijsman, C. E. Hack, and A. Sturk, Circulation 96, 3534 (1997).

19. R. A. Preston, W. Jy, J. J. Jimenez, L. M. Mauro, L. L. Horstman, M. Valle, G. Aime, and Y. S. Ahn, Hypertension 41, 211 (2003).

20. J. Alijotas-Reig, C. Palacio-Garcia, I. Farran-Codina, C. Zarzoso, L. Cabero-Roura, and M. Vilardell-Tarres, Am. J. Reprod. Rmmunol. 66, 199 (2011).

21. A. Aharon and B. Brenner, Thromb. Res. 127, 67 (2011).

22. B. Fevrier and G. Raposo, Curr. Opin. Cell Biol. 16, 415 (2004).

23. J. M. Freyssinet and F. Dignat-George, J. Thromb. Haemost. 3, 613 (2005).

24. W. Jy, L. L. Horstman, J. J. Jimenez, and Y. S. Ahn, J. Thromb. Haemost. 2, 1842 (2004).

25. A. K. Enjeti, L. F. Lincz, and M. Seldon, Semin. Thromb. Hemost 33, 771 (2007).

26. Y. Yuana, T. H. Oosterkamp, S. Bahatyrova, B. Ashcroft, P. Garcia Rodriguez, R. M. Bertina, and S. Osanto, J. Thromb. Haemost. 8, 315 (2010).

27. A. Habib, C. Kunzelmann, W. Shamseddeen, F. Zobairi, J. M. Freyssinet, and A. Taher, Haematologica. 93, 941 (2008).

28. V. Filipe, A. Hawe, and W. Jiskoot, Pharm. Res. 27, 796 (2010).

29. B. A. Ashcroft, J. De Sonneville, Y. Yuana, S. Osanto, R. Bertina, M. E. Kuil, and T. H. Oosterkamp, Biomed. Microdevices 14, 641 (2012).

30. C. Thery, S. Amigorena, G. Raposo, and A. Clayton, Current Protocols in Cell Biology, John Wiley \& Sons, Inc., New York (2001).

31. S. Nomura, A. Shouzu, K. Taomoto, Y. Togane, S. Goto, Y. Ozaki, S. Uchiyama, and Y. Ikeda, J. Atheroscler. Thromb. 16, 878 (2009).

Received: 6 September 2015. Accepted: 15 November 2015. 